\title{
Infering and Calibrating Triadic Closure in a Dynamic Network
}

\author{
Alexander V. Mantzaris and Desmond J. Higham
}

\begin{abstract}
In the social sciences, the hypothesis of triadic closure contends that new links in a social contact network arise preferentially between those who currently share neighbours. Here, in a proof-of-principle study, we show how to calibrate a recently proposed evolving network model to time-dependent connectivity data. The probabilistic edge birth rate in the model contains a triadic closure term, so we are also able to assess statistically the evidence for this effect. The approach is shown to work on data generated synthetically from the model. We then apply this methodology to some real, large-scale data that records the build up of connections in a business-related social networking site, and find evidence for triadic closure.
\end{abstract}

\section{Motivation}

Many modern application areas give rise to patterns of connectivity that change over time [9]. Examples include mobile telecommunication, on-line trading, smartmetering, massive multiplayer online gaming and online social networking. Information such as 'who called who', 'who tweeted who', 'who FaceTimed who', and 'people who bought his book also bought ...' is naturally evolving over time and cannot be fully exploited through a static representation as a single time-average or snapshot. These emerging, data-rich disciplines generate large, highly-resolved network sequences that demand new models and computational tools.

This work focuses on the use of a mathematical model to describe the microscale, transient dynamics. The model, from [5], is mechanistic, incorporating the triadic closure effect that many social scientists believe to be a key driving force behind social interactions. We show how a likelihood approach can be used to calibrate the model, thereby allowing the triadic closure hypothesis to be tested statistically on real data.

Department of Mathematics and Statistics, University of Strathclyde, Glasgow, UK. e-mail: alexander.mantzaris@strath.ac.uk 
The manuscript is organised as follows. In Section 2 we introduce the triadic closure concept and discuss some relevant work in the area. In Section 3 we describe the model from [5] and illustrate its use. Section 4 then explains how the likelihood - the probability of observing the microscale, edge by edge, data given a set of model parameters - can be computed and used to perform statistical inference. To illustrate the idea, we generate synthetic data from the model and reverse engineer the model parameters. In Section 5 we then apply these ideas to a set of online social interaction data. Section 6 gives a summary and points to future work.

\section{Background}

The concept of triadic closure can be traced back to the work of the sociologist Georg Simmel in the early 1900s, and was popularized by the influential article [4]. It is a key motivation for the use of clustering coefficients [3, 15] to summarize network properties. The basic principle can be summarized as $[2,16]$

Triadic Closure, part 1: If two unconnected people in a social network have a friend in common, then there is an increased likelihood that they will become friends themselves at some point in the future.

As discussed in [2, Chapter 3], there are at least three convincing reasons why triadic closure might feature in the evolution of a social interaction network. If $\mathrm{B}$ and $\mathrm{C}$ are not currently friends, but share a common friend, $\mathrm{A}$, then a new link from $\mathrm{B}$ to $\mathrm{C}$ is more likely to arise than a link between an arbitrary pair, through

Opportunity: B and C both socialize with A and hence have more chance of meeting,

Trust: A can simultaneously vouch for both B and C,

Incentive: A may view the triadic friendship as less stressful or time-consuming to maintain than the separate pair of dyads, and hence encourage the B-C link.

Based on these points, we may also argue that each extra common friend shared by $\mathrm{B}$ and $\mathrm{C}$ will increase the chance of a future B-C link. Hence, we may slightly extend the triadic closure principle above:

Triadic Closure, part 2: The likelihood that two unconnected people in a social network will become friends themselves at some point in the future increases with the number of friends they share in common.

The digital age has given rise to large scale human interaction data sets, making it feasible to test the hypothesis that changes in connectivity are driven by triadic closure. For example, Leskovec et al. [12] studied four large online social network datasets and found that "most new edges span very short distances, typically closing triangles." Mislove et al. [14] monitored the growth of the Flickr network and found that $80 \%$ of new links "connected users that were only two hops apart, meaning that the destination user was a friend-of-a-friend of the source user before the new link was created". Szell et al. [17] measured transition rates between dyadic and triadic 
structures for positive and negative social interactions in a massive multiplayer online game and found "overrepresentation (underrepresentation) of complete triads in networks of positive ties, and vice versa for networks of negative ties."

Networks in neuroscience have also been observed to have an overabundance of triangles, with respect to both anatomical and functional connectivity $[1,7,13]$.

In this work we aim to go beyond the realm of simply recording the incidence of network triangles by combining ideas from applied mathematics and applied statistics. Given network data, we wish to calibrate an appropriate mechanistic model of network evolution and simultaneously quantify the statistical evidence in favour of triadic closure.

The closest previous work to ours is perhaps [12], where stochastic models incorporating triadic closure were proposed and tested in a microscale/likelihood setting. To understand the class of models from [12], consider the node $u$ in Figure 1 (which is based on Figure 6 from [12]). The triangulation stage adds a link to a node, $w$, that is two hops away from $u$. This is done by first choosing one of $u$ 's neighbours, $v$, according to one of the five following rules

random: uniformly at random,

degree: proportional to some power of the degree of node $v$,

common friends: proportional to the number of common friends shared by nodes $u$ and $v$,

last time: proportional to some power of the time that has elapsed since $v$ last created an edge,

comlast: proportional to the product of (a) the number of common friends shared by nodes $u$ and $v$, and (b) the time that has elapsed since $v$ last created an edge, raised to some power.

Similarly, any of these five rules can be used to chose a neighbour, $w$, of $v$. The new link is then inserted between $u$ and $w$ in order to create a triad. This gives a total 25 different models, which were tested in [12] on real data in a likelihood setting. The authors concluded that the random-random model (choose $v$ uniformly from the neighbours of $u$ and then choose $w$ uniformly from the neighbours of $v$ ) gives a good compromise between accuracy and simplicity.

In this work, we consider a recent stochastic triadic closure model from [5], based on the general methodology of [6]. A key difference from the versions discussed above is that this model triangulates by directly choosing nodes $w$ that are two hops from $u$, with a bias that is proportional to the number of common neighboursdirectly reflecting part 2 of the triadic closure principle. Advantages of this modelling approach are that:

- A single parameter, $\varepsilon$, is used to quantify the strength of the triadic closure effect. The case $\varepsilon=0$ corresponds to no preference for triadic closure (edges appear uniformly at some basal rate). Hence, we have a nested pair of models and can test whether there is statistically significant evidence for triadic closure in the data.

- The modeling framework is sufficiently simple to allow a mean-field approximation for the evolution of the edge density. The mean-field approximation involves 


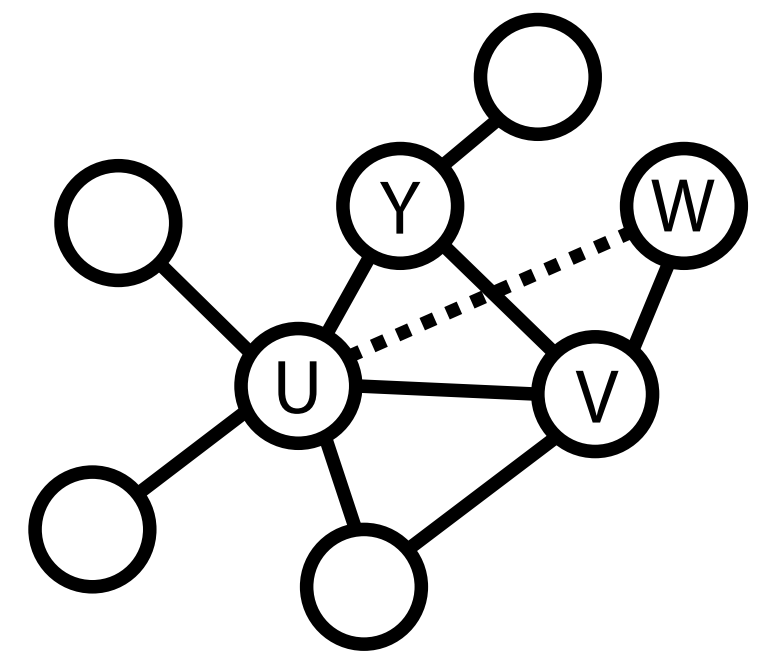

Fig. 1: Depiction of the triangulation process, based on Figure 6 from [12]. A new edge is produced, as shown by the dashed line. This dashed edge creates a triad closure between nodes $\mathrm{U}, \mathrm{V}$, and $\mathrm{W}$.

the same model parameters, and hence this macroscopic summary data can be used when a full likelihood computation at the microscale is not feasible. We exploit this feature in section 5.

\section{The Triadic Closure Model}

We suppose that a fixed set of $N$ nodes have a connectivity structure that may change at discrete, uniformly spaced, time points $t_{0}<t_{1}<\ldots<t_{K}$. We let $A_{k}$ denote the adjacency matrix for the network at time $t_{k}$ and assume that the networks are unweighted and undirected without self-loops, so each $A_{k} \in \mathbb{R}^{N \times N}$ has $a_{i j}=a_{j i}=1$ if there is an edge from node $i$ to node $j$ at time $t_{k}$ and has $a_{i j}=a_{j i}=0$ otherwise, with all $a_{i i}=0$.

The triadic closure model in [5] involves two matrix-valued functions of the current state, $\omega\left(A_{k}\right)$, and $\alpha\left(A_{k}\right)$, giving the edge death and edge birth probabilities, respectively. The death probability takes the simple form

$$
\left(\omega\left(A_{k}\right)\right)_{i j} \equiv \tilde{\omega}, \quad \text { for some } \tilde{\omega} \in(0,1) .
$$

The birth probability is defined as

$$
\left(\alpha\left(A_{k}\right)\right)_{i j}=\delta+\varepsilon\left(A_{k}^{2}\right)_{i j}
$$


for some constants $\delta$ and $\varepsilon$. We impose $0<\varepsilon(N-2)<1-\delta$ to ensure that the birth probability lies between 0 and 1 .

Given the time $t_{k}$ network, $A_{k}$, if there is currently no edge from $i$ to $j$, then the birth probability specifies the chance that the edge will emerge at time $t_{k+1}$. Similarly, if $i$ and $j$ are connected in $A_{k}$, the death probability specifies the chance that the edge will disappear at at time $t_{k+1}$. Conditioned on $A_{k}$, all such edge events are taken to be independent. More precisely, the model takes the form of a discrete time Markov chain over the state space of all binary, symmetric networks with $N$ nodes, and given $A_{0}$ we may simulate a path of the chain as follows

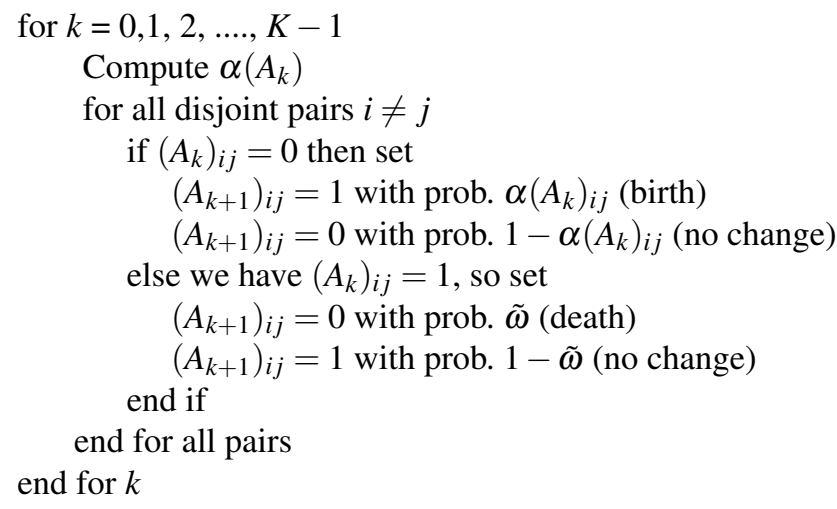

To understand the form of the birth probability (2), we note that the factor $\left(A_{k}^{2}\right)_{i j}$ counts the number of neighbours shared by nodes $i$ and $j$ at time $t_{k}$. Hence the overall birth probability is given by combining

- a basal level, $\delta$, and

- a triadic closure term that is proportional to the number of new triangles the edge would create. Here $\varepsilon$ controls the strength of the triadic closure effect.

A mean-field approximation for the evolution of a macroscopic quantity, the edge density

$$
\widehat{p}_{k}:=\frac{1}{N(N-1) / 2} \sum \sum_{i>j}\left(A_{k}\right)_{i j}
$$

was proposed in [5] and found to match well with real simulations. This mean-field approximation takes the form

$$
p_{k+1}=(1-\tilde{\omega}) p_{k}+\left(1-p_{k}\right)\left(\delta+\varepsilon(N-2) p_{k}^{2}\right) .
$$

An interesting feature of the iteration (4) is that there are generically three steady states; that is, values $p^{\star}$ such that $p_{k+1}=p_{k}=p^{\star}$ solves (4), with only the outer two being stable. Simulations showed that both stable states could be observed in practice, and in particular for initial networks $A_{0}$ close to the intermediate, unstable steady state, the density along a path evolves to one of the two stable values in an unpredictable manner that depends on the precise micro-level detail. 
To illustrate the model, and also to provide some data for the inference computations in the next section, we now show some network sequences generated by the model. In each case we used 100 nodes and 100 time points, and the initial network, $A_{0}$, was a sample of a classical Erdös-Rényi random graph with expected edge density of $p$; we denote this by $A_{0}=\operatorname{ER}(p)$.

Data Set A: $\delta=0.0004, \tilde{\omega}=0.01, \varepsilon=0, A_{0}=\mathrm{ER}(0.5)$.

In this case there is no triadic closure-edges appear and disappear uniformly at random. The mean-field equation (4) collapses to a linear iteration with a single, stable steady state at $p^{\star}=0.0385$. Figure 2 shows the adjacency matrix at selected time points, along with the edge density as a function of time. The images of the adjacency matrix put a black square where the value is zero and a white square where the value is one. To show the relevance of the mean-field approximation, Figure 3 illustrates what happens when the path is followed for a longer period; we used 1000 time points. The mean-field approximation from (4) is superimposed over the edge density plots. Here the observed edge density at the final time point is 0.0356 .

Data Set B: $\delta=0.0004, \widetilde{\omega}=0.01, \varepsilon=0.0005, A_{0}=\operatorname{ER}(0.5)$.

Here, the mean-field iteration has steady states 0.7215 (stable), 0.2291 (unstable) and 0.0494 (stable). Using $A_{0}=\operatorname{ER}(0.5)$ starts the paths closest to the denser of the two stable macro-states. Figure 4 shows some network snapshots along with the edge density. A longer time interval is used in Figure 5 in order to confirm the relevance of the $p^{\star}=0.7215$ steady state.

Data Set C: $\delta=0.0004, \tilde{\omega}=0.01, \varepsilon=0.0005, A_{0}=\mathrm{ER}(0.1)$.

Here, we use the same model parameters as in Data Set B, but start with a less dense initial network. Figure 6 shows network snapshots and edge density, and Figure 7 runs over a longer time period. In this case, the edge density is attracted to the other stable steady state at $p^{\star}=0.0494$.

\section{Likelihood and Inference}

The probabilistic nature of the model produces a natural likelihood function to feed into a calibration and inference framework. This section explains the details and tests out the idea on the data sets from Section 3.

Given network data $A_{0}, A_{1}, \ldots, A_{k}$, that is, up to time $t_{k}$, because the model satisfies the Markov property the likelikood of observing a network $A_{k+1}$ at time $t_{k+1}$ depends only on $A_{k}$ and is given by

$\mathscr{L}\left(A_{k+1} \mid A_{k}\right)=\prod_{\text {Remain alive }}(1-\tilde{\omega}) \times \prod_{\text {Become alive }} \alpha\left(A_{k}\right)_{i j} \times \prod_{\text {Remain dead }}(1-\alpha)\left(A_{k}\right)_{i j} \times \prod_{\text {Become dead }} \tilde{\omega}_{i j}$.

Here the products are over appropriate ordered edge pairs; that is, $(i, j)$ with $i<j$; so for example, "Remain alive" denotes $(i, j)$ for which both $\left(A_{k}\right)_{i j}=1$ and $\left(A_{k+1}\right)_{i j}=$ 1. 

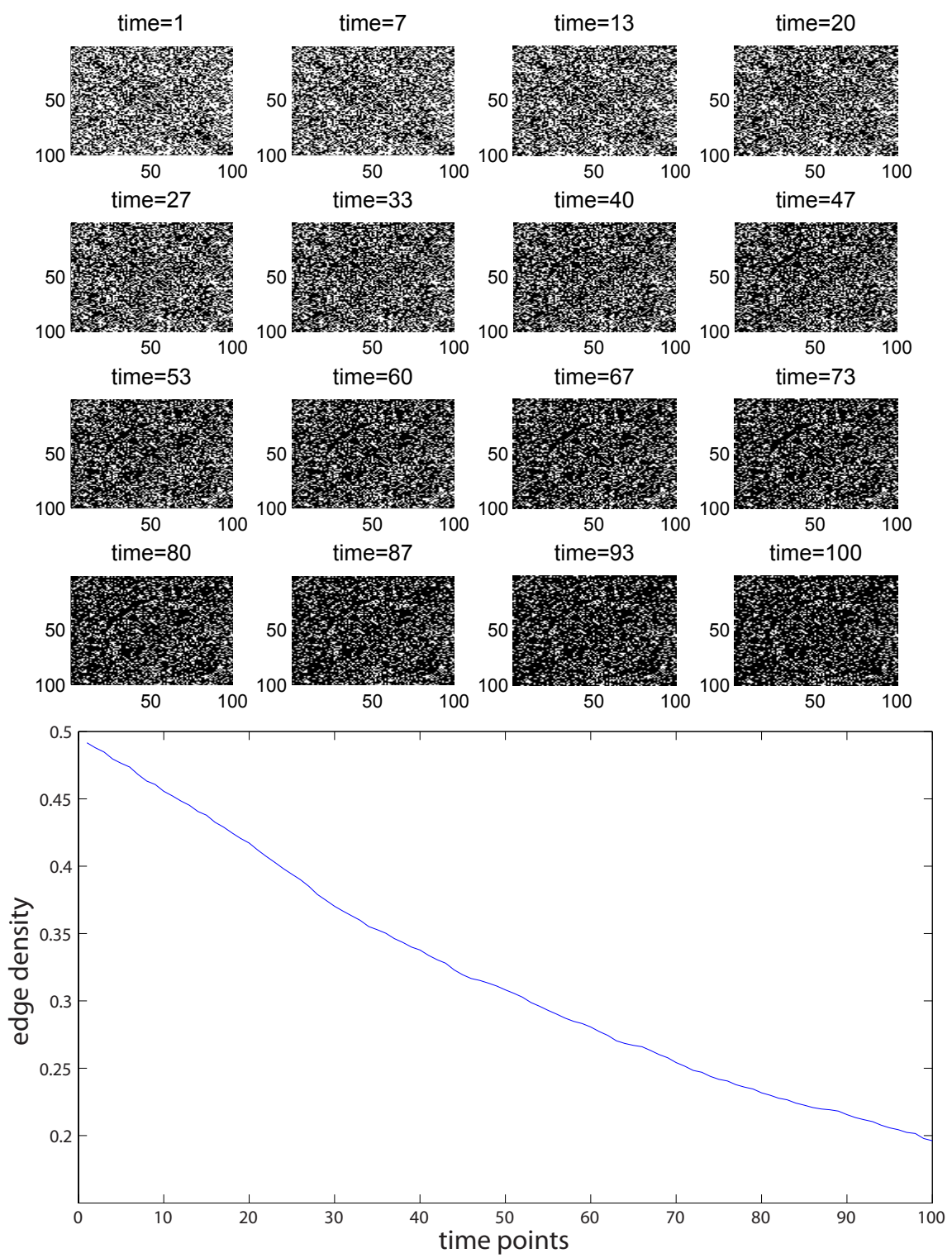

Fig. 2: Data set A. Upper: the adjacency matrix at selected time points. Lower: edge density as a function of time. 

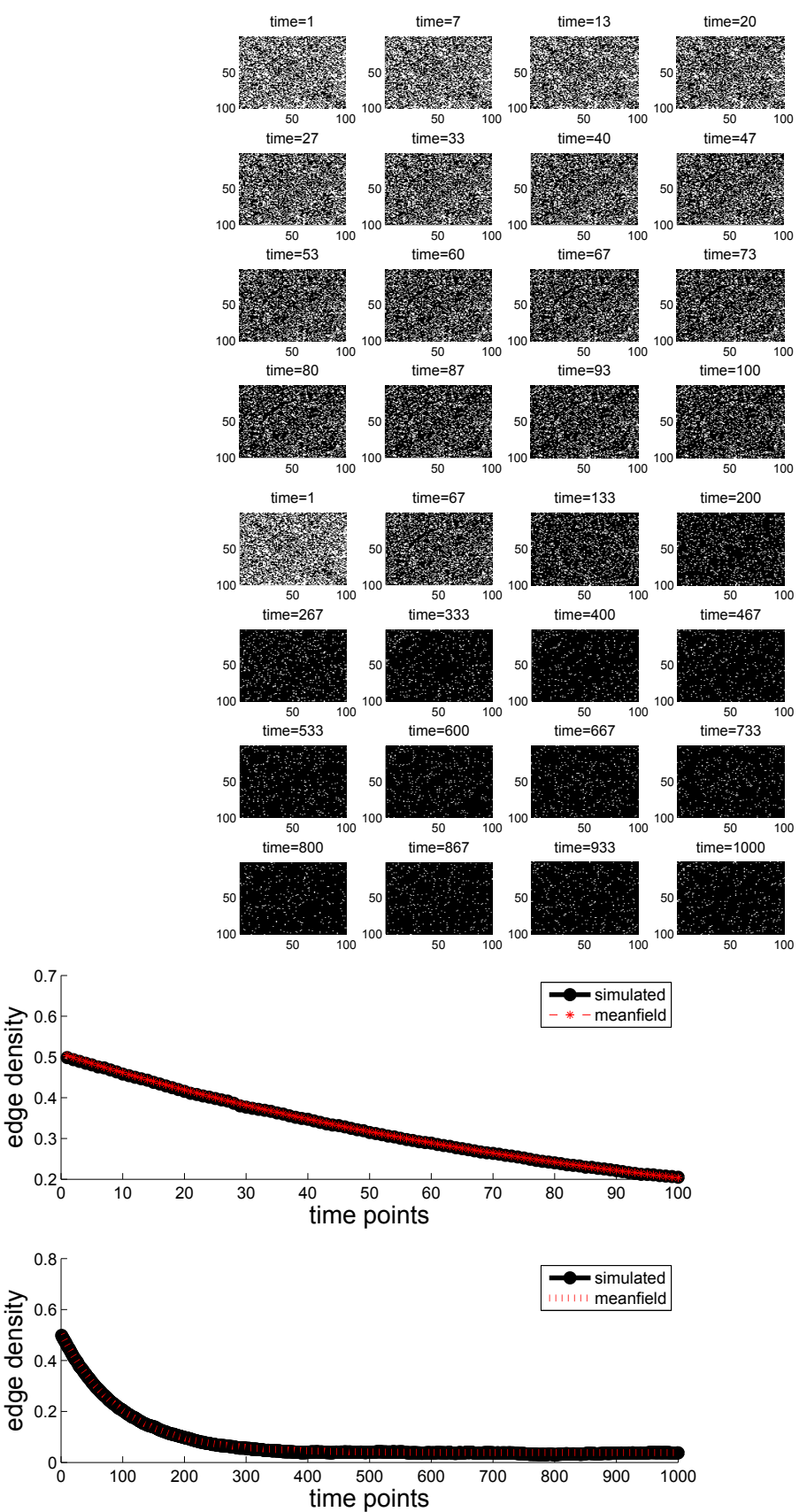

Fig. 3: Extended version of Data set A. Upper: the adjacency matrix at selected times points up to time 1000. Lower: edge density up to time 100 and time 1000 . 

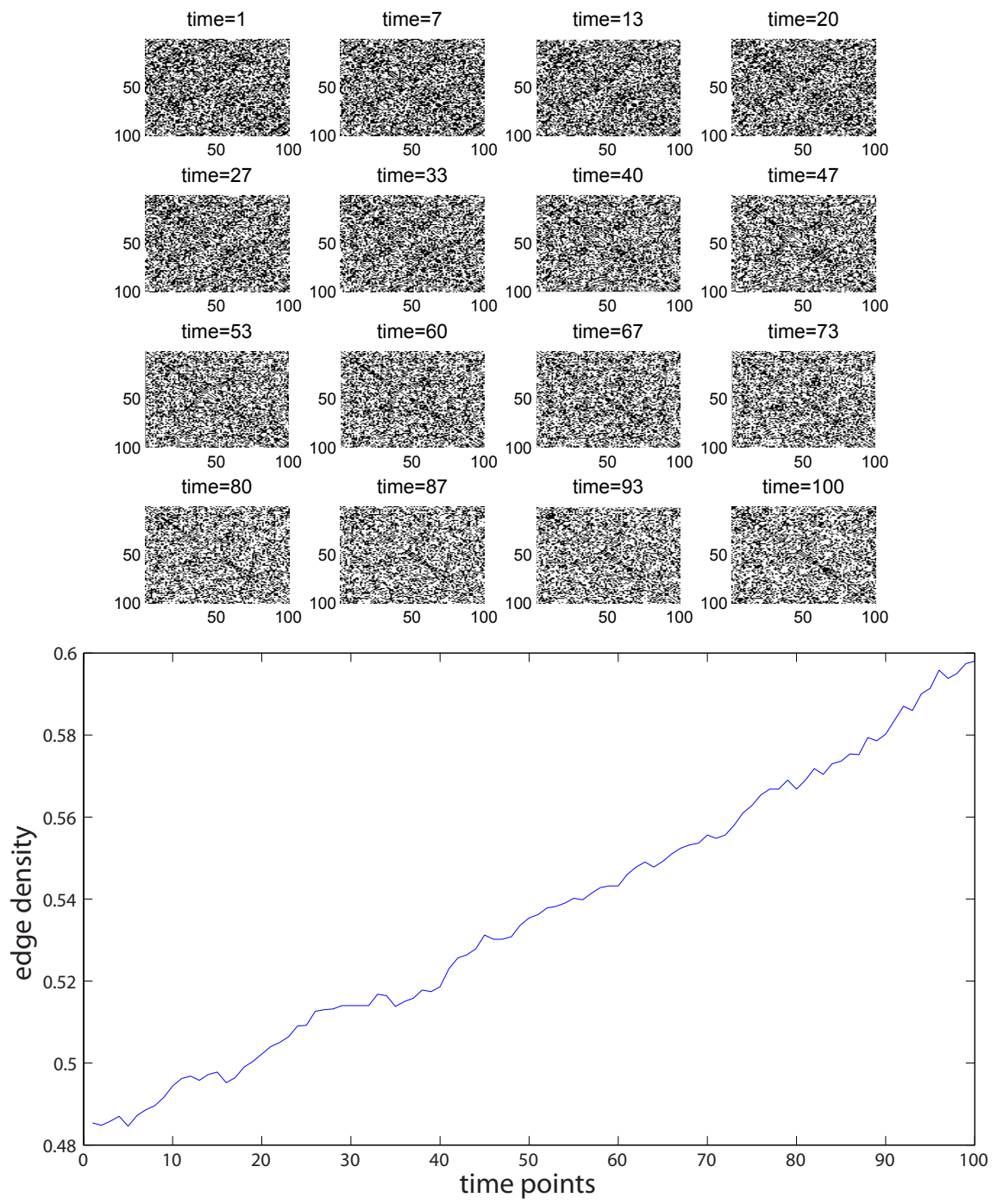

Fig. 4: Data set B. Upper: the adjacency matrix at selected time points. Lower: edge density as a function of time. 

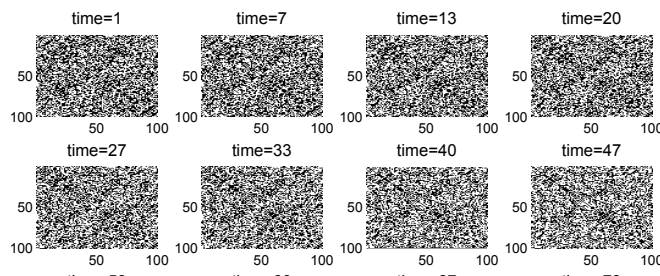

time $=53$

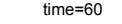

time $=67$

50
+100
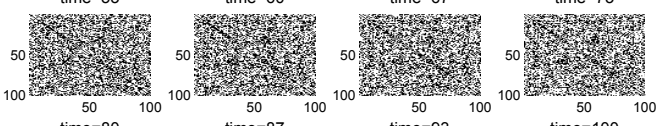

time $=80$

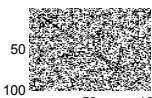

time $=87$

time $=93$
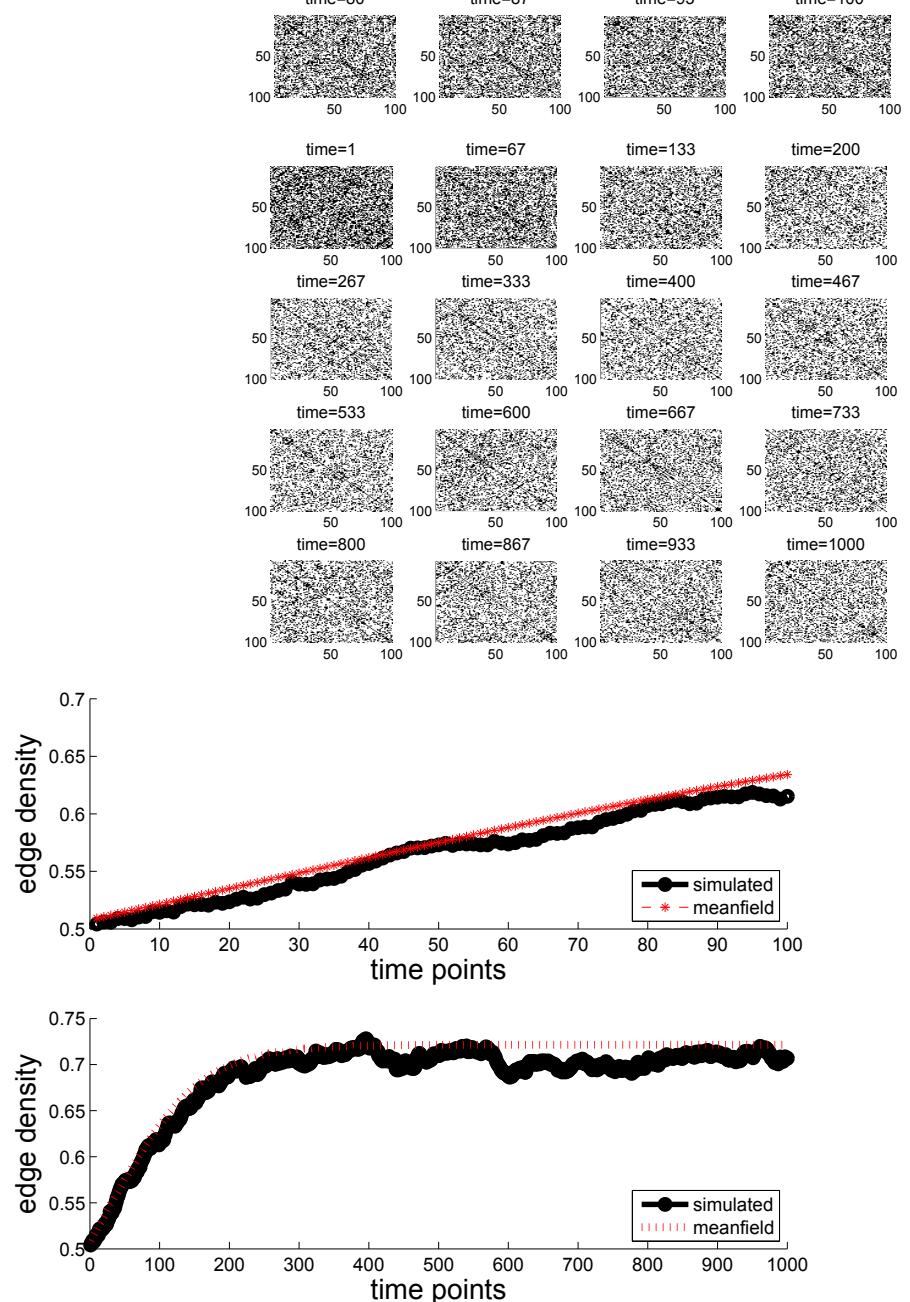

Fig. 5: Extended version of Data set B. Upper: the adjacency matrix at selected times points up to time 1000. Lower: edge density up to time 100 and time 1000. 

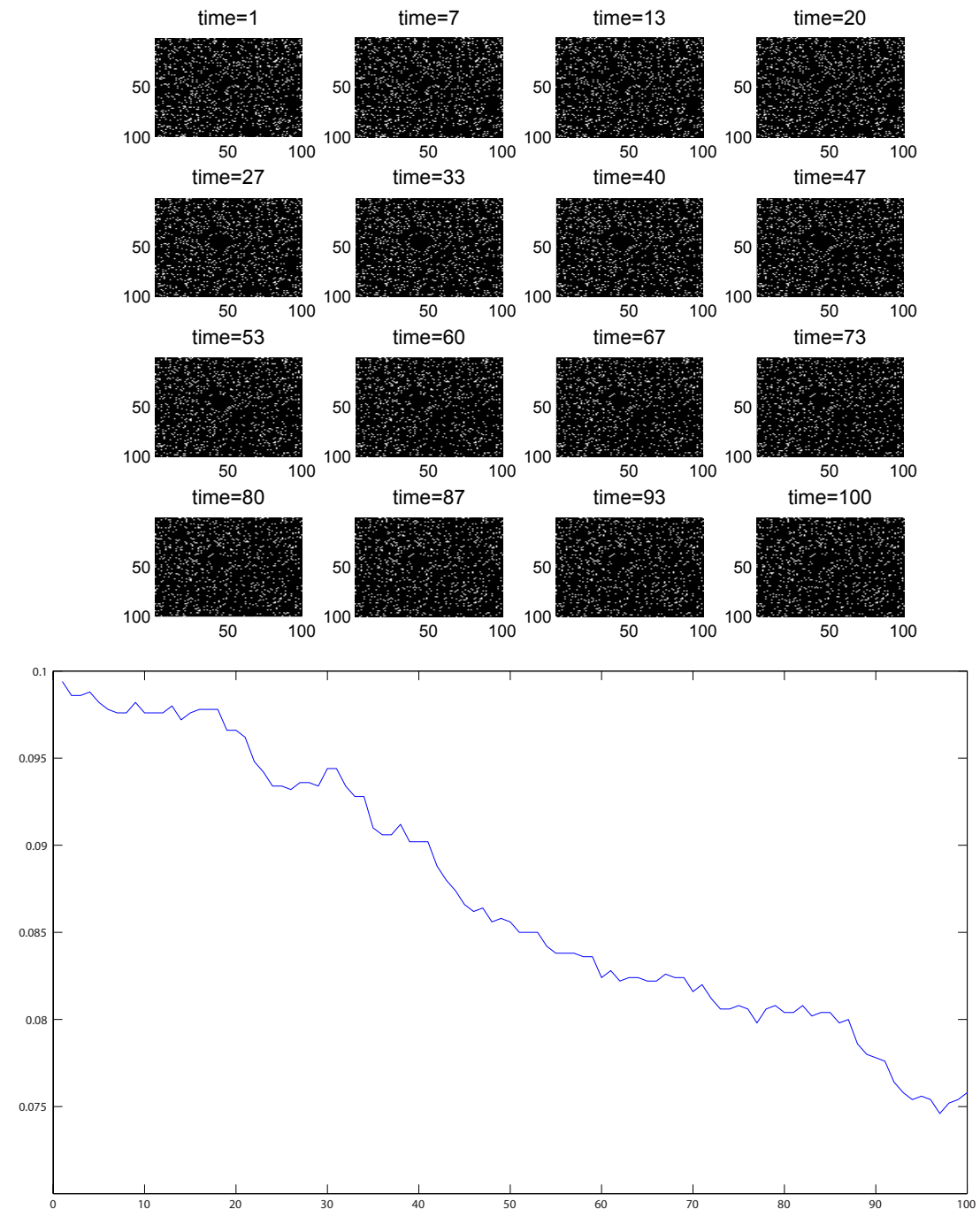

Fig. 6: Data set C. Upper: the adjacency matrix at selected time points. Lower: edge density as a function of time.

Now suppose that the model parameters and initial network $A_{0}$ are fixed. Any observed network sequence $A_{1}, A_{2}, \ldots, A_{K}$ then has likelihood

$$
\mathscr{L}\left(A_{1} \mid A_{0}\right) \times \mathscr{L}\left(A_{2} \mid A_{1}\right) \times \cdots \times \mathscr{L}\left(A_{K} \mid A_{K-1}\right) .
$$

The parameters which maximise the likelihood of a model may then be calculated. In this work we use straightforward grid searches over appropriate parameter 

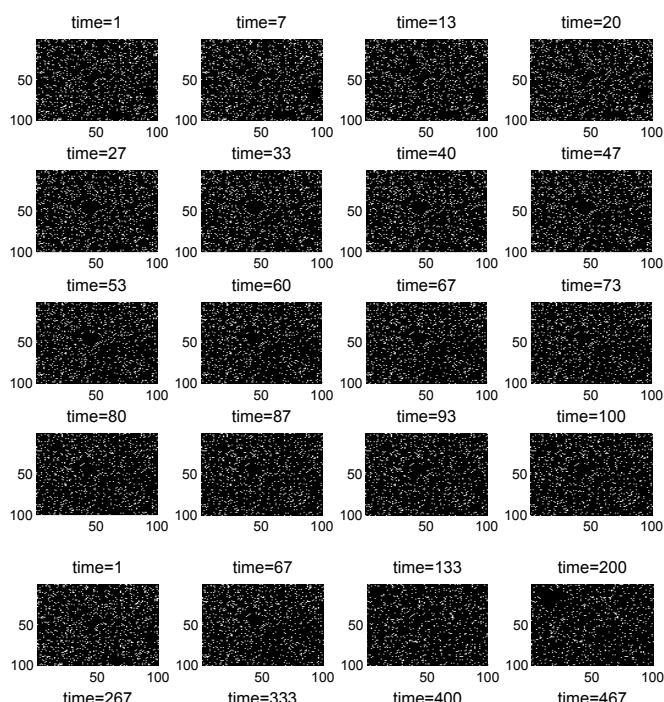

time $=67$

time $=133$
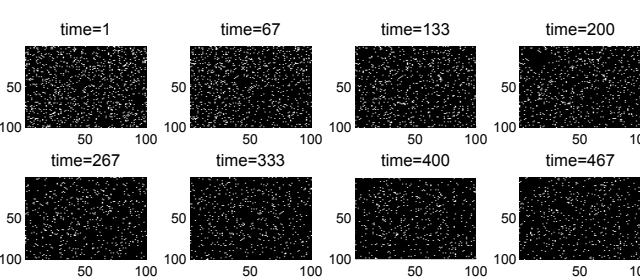

time $=400$
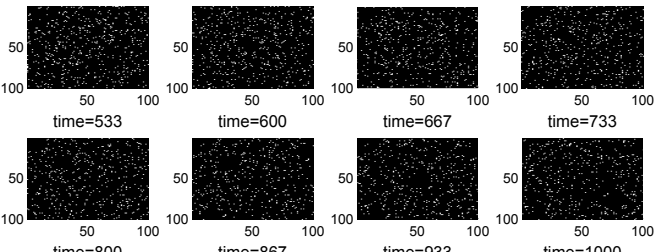

time $=600$
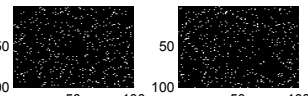

time $=933$
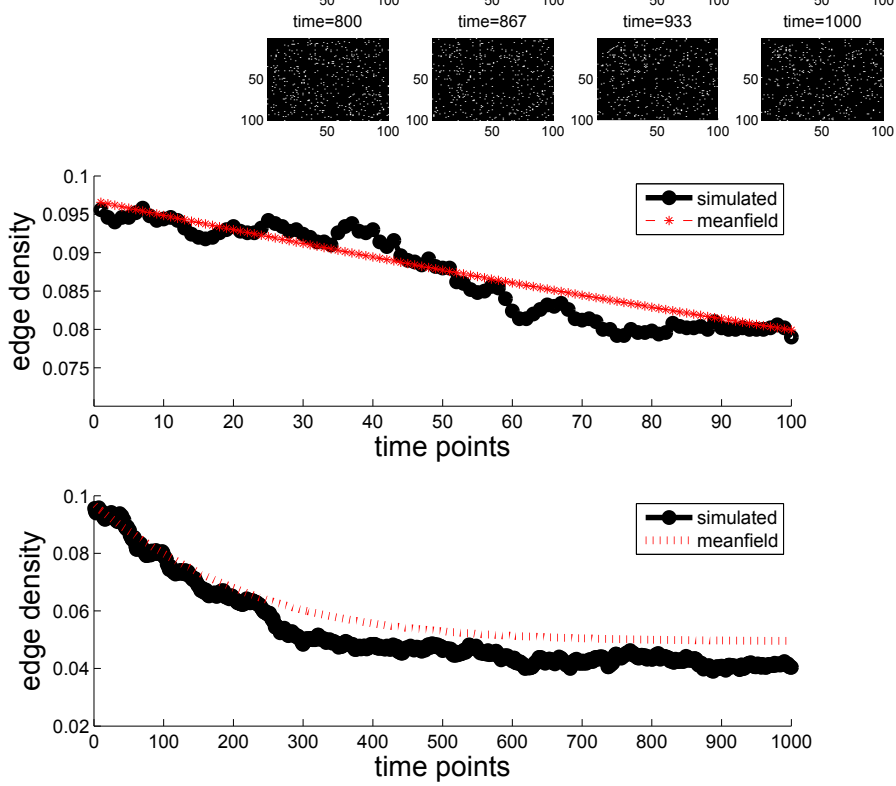

Fig. 7: Extended version of Data set C. Upper: the adjacency matrix at selected times points up to time 1000. Lower: edge density up to time 100 and time 1000 . 
ranges. We focus here on the constrained model, where $\varepsilon$ is fixed at zero, so no triad closure effect is present, and the unconstrained model, where $\varepsilon$ is a model parameter. The constrained model is therefore nested within the unconstrained model, which makes the application of the likelihood ratio test [18] suitable for model comparison. The likelihood ratio test value, $D$, is then used to compute a p-value for rejecting the null model, which is the constrained model. The value $D$ and the difference in degrees of freedom in the unconstrained and constrained model are used as parameters for the chi-squared distribution. This allows us to compute a p-value for rejecting the null hypothesis. We take a threshold of 0.01 .

The Akaike information criterion (AIC), [8], is also used here for model selection. AIC is founded in information theory. The application of AIC reinforces the results of the likelihood ratio test.

\section{Inference for Data Set A:}

Here we performed a grid search of the likelihood over $\delta=0.0001: 0.0001: 0.0006$ and $\tilde{\omega}=0.0005: 0.005: 0.025$ for the constrained model. For the unconstrained model we also used $\varepsilon=0.0000: 0.0001: 0.001$.

The search for the constrained model gave $\alpha_{\max }=0.0004$ and $\omega_{\max }=0.0105$ with a $\log$ likelihood of -10182.0284 . For the unconstrained model, we obtained $\alpha_{\max }=0.0004, \omega_{\max }=0.0105$ and $\varepsilon_{\max }=0$, with a log likelihood of -10182.0284 . Hence, the extra freedom offered by $\varepsilon$ was clearly not relevant for this data set. The $\log$ likelihood ratio test therefore gives $D=0$, favouring the constrained model. The marginal for $\varepsilon$ is shown in Figure 8. We can see the decay away from the true value of zero. AIC also prefers the model without triadic closure.

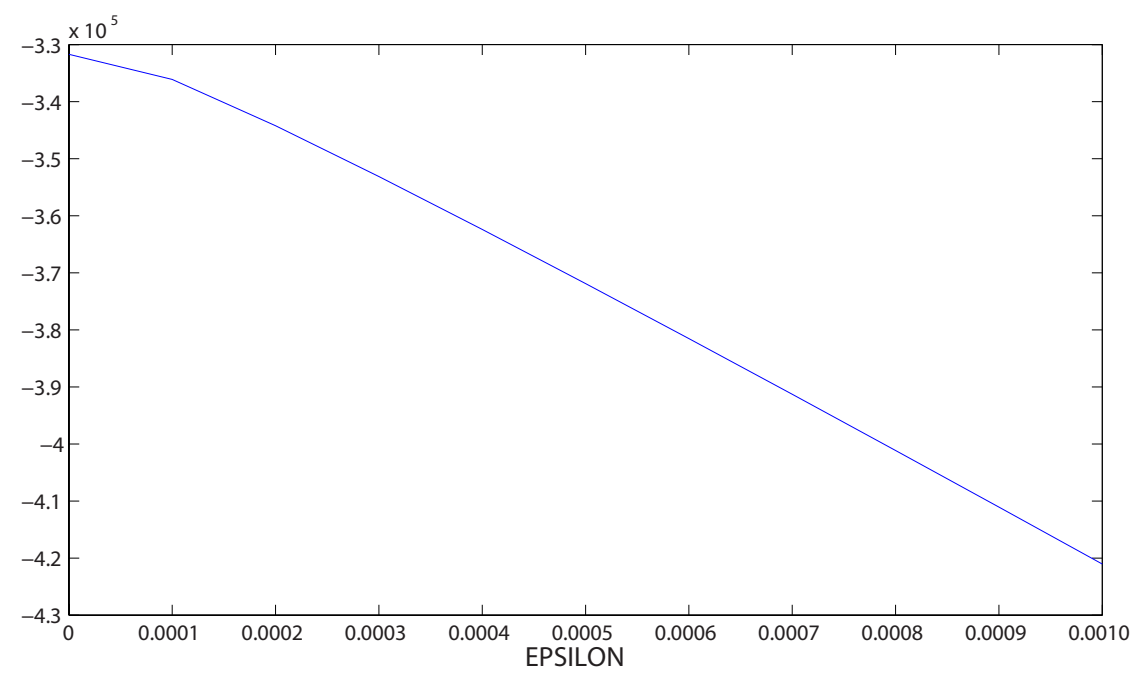

Fig. 8: Marginal for $\varepsilon$ in Dataset A. 
Inference for Data Set B:

Here we searched over $\alpha=0.0001: 0.0001: 0.0006$ and $\omega=0.0005: 0.005: 0.025$ in the constrained model and also $\varepsilon=0.0000: 0.0001: 0.001$ in the unconstrained model. The likelihood for the constrained model is maximized at $\alpha_{\max }=0.0006$ and $\omega_{\max }=0.0105$ with $\log$ likelihood of -38633.7891 . For the unconstrained model, $\alpha_{\max }=0.0001, \omega_{\max }=0.0105$ and $\varepsilon_{\max }=0.0005$ with $\log$ likelihood -31477.9615 . We note that the constrained model has a higher birth rate to compensate for the lack of triangulation. The $\log$ likelihood ratio test value is $D=14311.6553$, so the test result is 1 , favouring the triad closure model. AIC chooses the model that has triad closure as well. The marginal of $\varepsilon$, shown in Figure 9, has a clear peak around the value of 0.0005 that was used to generate the data.

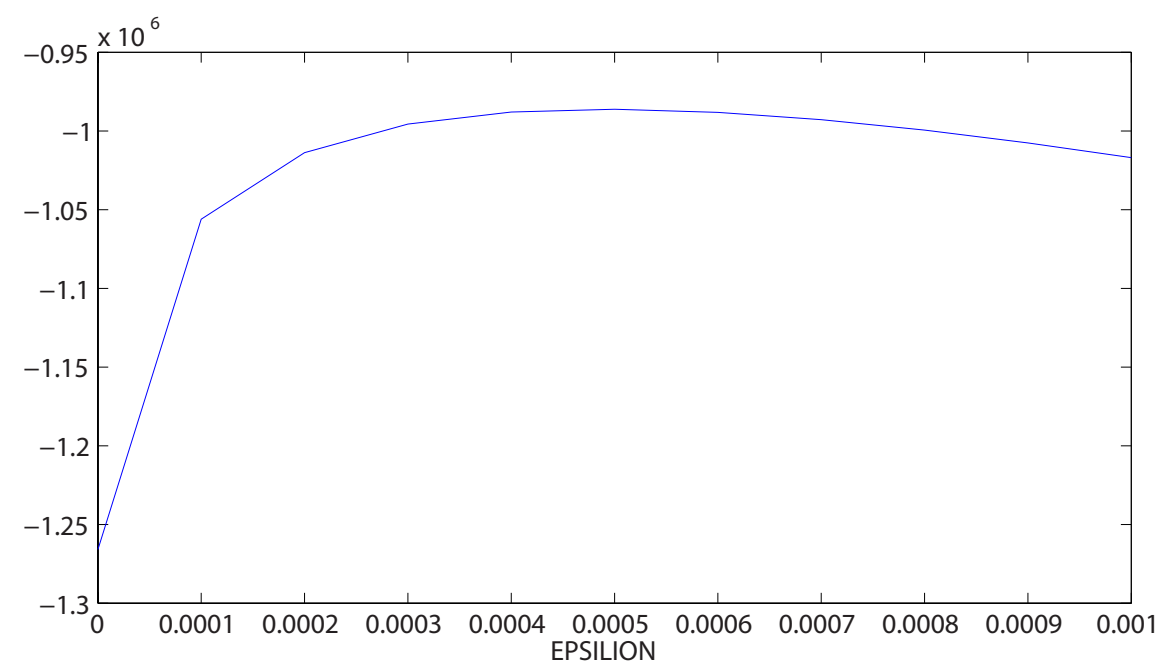

Fig. 9: Marginal for $\varepsilon$ in Dataset B.

\section{Inference for Data Set C:}

For the constrained model the grid search took place over $\alpha=0.0001: 0.0001$ : 0.0006 and $\omega=0.0005: 0.005: 0.025$ and for the unconstrained model we also used $\varepsilon=0.0000: 0.0001: 0.001$. The constrained model produced $\alpha_{\max }=0.0006$ and $\omega_{\max }=0.0105$ with $\log$ likelihood of -5136.5503 . For the unconstrained model we have $\alpha_{\max }=0.0004, \omega_{\max }=0.0105$ and $\varepsilon_{\max }=0.0005$ with $\log$ likelihood of -5089.8203 . As for Data set B, the constrained model has a higher birth rate to balance the lack of triangulation. The log likelihood ratio test value is $D=93.4599$, with a test result of 1 , favouring the triad closure model. As well, AIC chooses the model with triad closure. The marginal of $\varepsilon$ is shown in Figure 10, and, as in Data set $\mathrm{B}$, we see a peak around the value of 0.0005 .

Based on these results, we conclude that the likelihood-based inference approach can produce meaningful results on this type of data. 


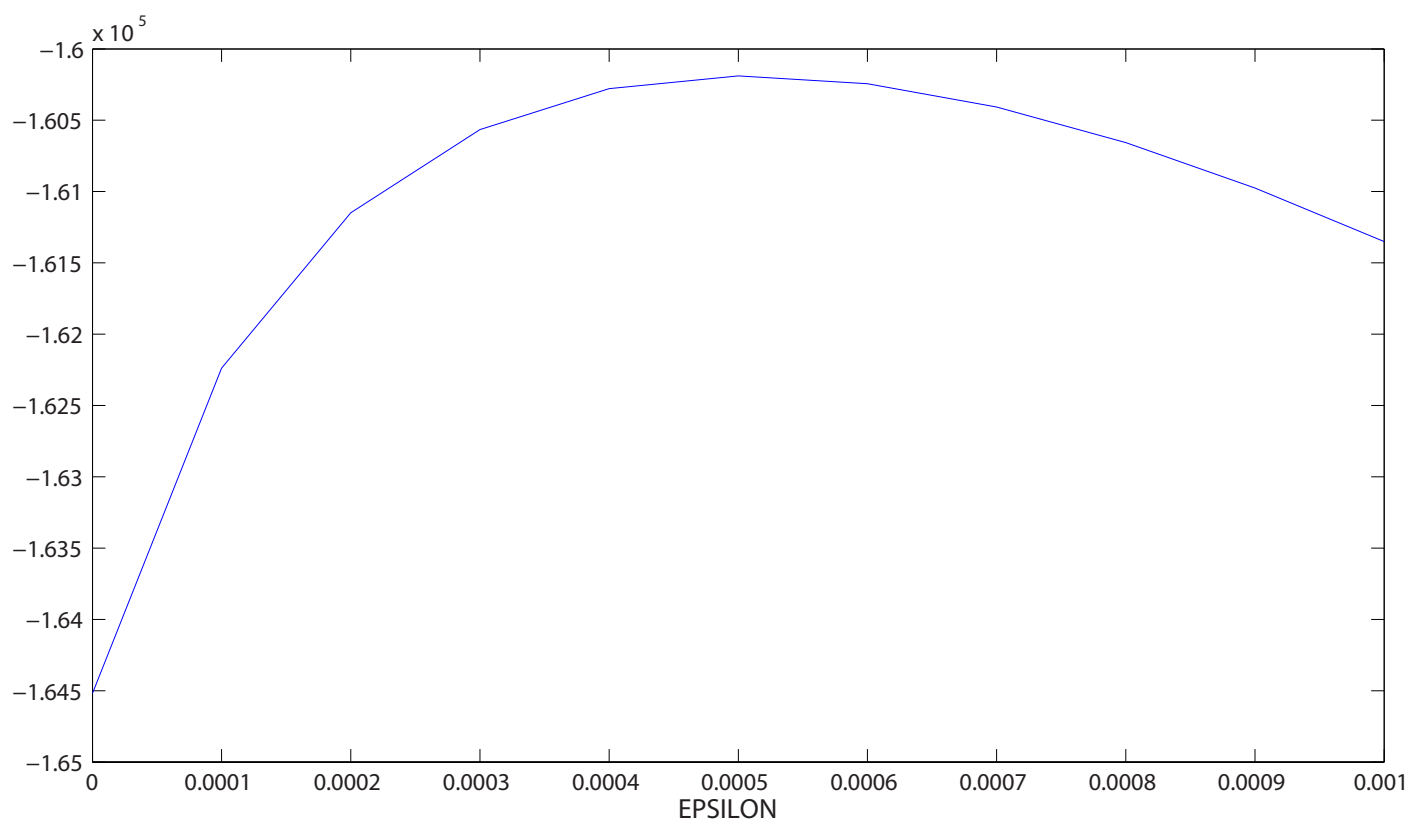

Fig. 10: Marginal for epsilon for Data set C.

\section{On-Line Social Network Data}

We now apply this methodology to online social network data from [10] that relates to the Wealink (http://www . wealink.com/) social networking site for professionals in China. In this data set, new users (nodes) join the network over time, and a new link can be established between any pair of current users. The raw data consists of triples of the form $(i, j, t)$, indicating that a link has been created between nodes $i$ and $j$ at time stamp $t$. There are 26,817,840 time stamps, measured in seconds, and they range from $t=1$ second to $72,711,888$ seconds, which is just over 841 days. The largest node id is 223,482 . For the purpose of calibration and model selection, we assume that all 223,482 nodes are present (i.e. available to form links) from the initial time point, and that all links are undirected. Hence we have symmetric adjacency matrices of dimension $N=223,482$. Edges do not disappear in this data set. Figure 11 shows how the edge density increases with time. The overall average rate of increase in Figure 11 is 0.0038 edges per second.

By construction, there is no edge death in this data set. Hence, we fix $\tilde{\omega}=0$. This leaves two parameters, the basal birth rate, $\delta$, and the triadic closure strength, $\varepsilon$. Just as we did in section 4 for the synthetic data, our aim is therefore to test whether there is evidence for a positive triadic closure strength. 


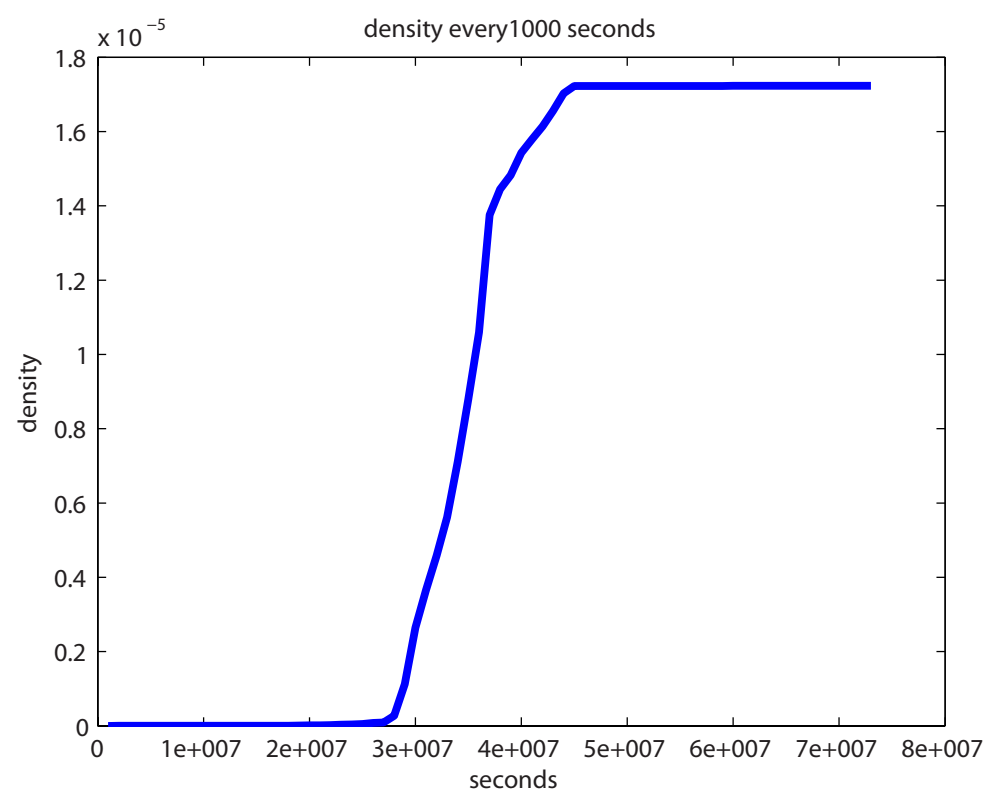

Fig. 11: Density of the Wealink online social network as a function of time in seconds.

Because of the large amount of data, we made a number of simplifications in order to reduce computational complexity to a feasible level. First, it is clear from Figure 11 that most activity takes place over a limited time period, and hence, for our experiments, we start with the network at time $3 \times 10^{7}$ seconds and continue until time $4 \times 10^{7}$ seconds. Then, rather than treating each event separately, we divide this period into 100 equally spaced time windows and construct an adjacency matrix for each. So each adjacency matrix records an element 1 in positions $(i, j)$ and $(j, i)$ if nodes $i$ and $j$ were already linked, or formed a link in the relevant time window, and it records a zero otherwise.

Due to the excessive cost of evaluating the likelihood, we first used the meanfield approximation (4) to get a feel for an appropriate range of parameter values. Figure 12 shows the edge density increasing over the 100 discrete time points. The red circles show the corresponding mean-field solution when we fix $\varepsilon=0$ and optimize in a mean-square sense over the remaining parameter, $\delta$; that is, given $p_{0}$, we consider the iteration

$$
p_{k+1}=p_{k}+\left(1-p_{k}\right) \delta
$$

and minimize the mean-square deviation between $\left\{p_{k}\right\}_{k>0}$ and the edge density from the data. This produces a value of $\delta=8.2059 \times 10^{-8}$ for the basal birth rate. The black crosses show the results when also allow the triadic closure strength to be 
nonzero; that is, we use

$$
p_{k+1}=p_{k}+\left(1-p_{k}\right)\left(\delta+\varepsilon(N-2) p_{k}^{2}\right) .
$$

Here, we found $\delta=9.53 \times 10^{-8}$ and $\varepsilon=7.32 \times 10^{-16}$.

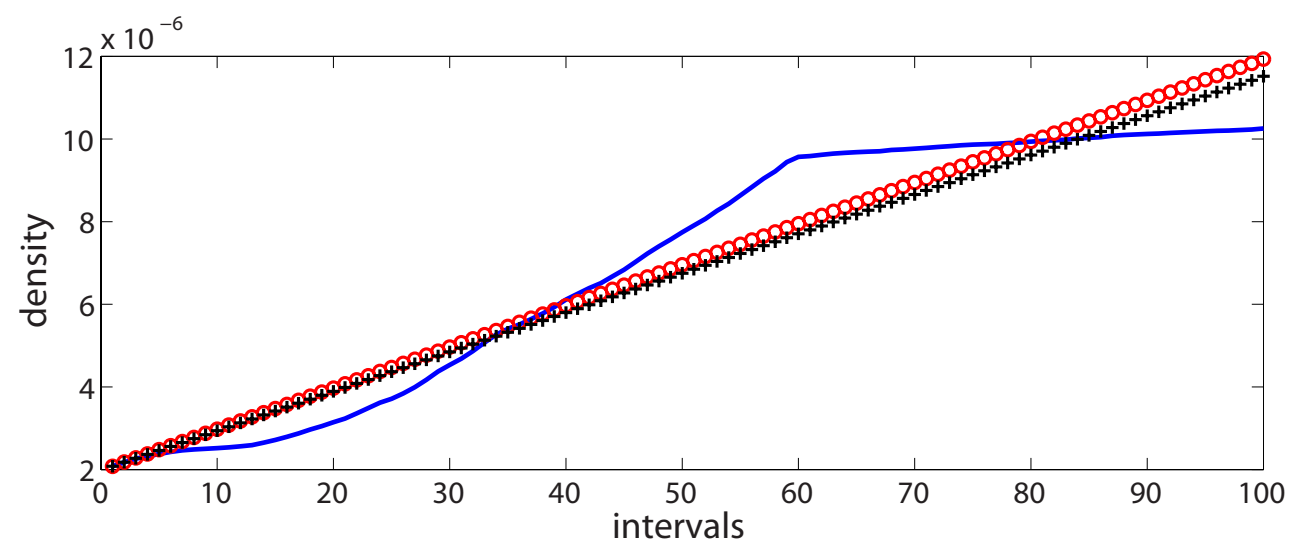

Fig. 12: Blue solid line: Edge density over the 100 discrete tme points used for the inference. Red circles: edge density from best mean-field fit with $\varepsilon$ fixed at zero and $\delta$ as a free parameter. Black crosses: edge density from best mean-field fit with both $\varepsilon$ and $\delta$ as free parameters.

Since our available computing power only permitted a full likelihood based calibration over one parameter, we then fixed $\delta=9.95 \times 10^{-8}$ and infered the triadic closure strength, $\varepsilon$, from the microscale data. Figure 13 shows the log likelihood as a function of $\varepsilon$. The best $\varepsilon$ value gives a $\log$ likelihood of $-1.58 \times 10^{8}$, whereas the $\varepsilon=0$ model produces $-1.97 \times 10^{8}$. We find that $D=7.81 \times 10^{7}$, and the triadic closure model is therefore chosen by the log likelihood ratio test. AIC also chooses the model with triadic closure.

\section{Summary}

Our aim in this work was to investigate calibration and inference issues for a stochastic microscale evolving network model. Edge dynamics in the model involve a term that quantifies a triadic closure effect-friends of friends tend to become friends. We showed that the presence of triadic closure can be inferred from a reasonable quantity of synthetic data, generated from the model. We then showed that there is statistical evidence for triadic closure in real data from a business-related social networking site. 


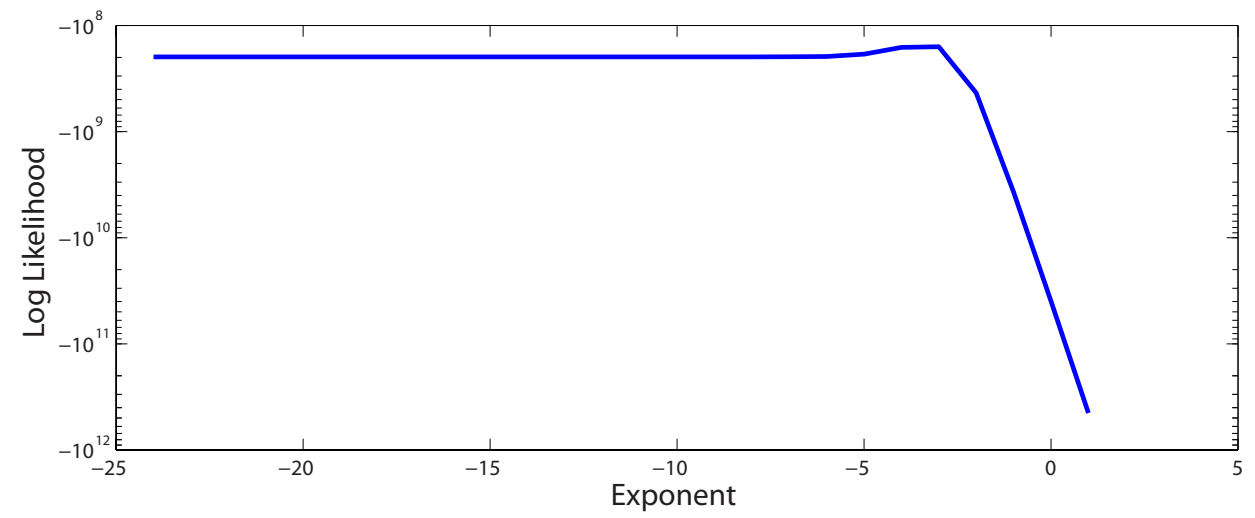

Fig. 13: Log likelihood of the triadic closure model as a function of triad closure strength, $\varepsilon$. The basal edge birth is fixed at $9.95 \times 10^{-8}$. The $\mathrm{x}$-axis shows the $\log$ base 10 values used for $\varepsilon$ in the search for the largest loglikelihood.

Although likelihood-based calibration and model comparison is conceptually straightforward for stochastic, Markov chain based, models of the type used here, the fundamental task is computationally challenging for large network sequences over long time periods. In this work, we were able to exploit a mean-field theory that describes the evolution of a macroscale quantity - the edge density-in terms of the model parameters.

There are many directions in which this type of work could be taken:

- other concepts from the social sciences that may determine network dynamics, such as homophily/heterophily, social distance and cultural drift $[2,11]$, could be quantified through mathematical models and then tested for and compared in real dynamic data sets,

- other types of interaction network, for example from telecommunication, online human behaviour and e-business, could be calibrated, compared and categorized,

- more sophisticated, customized strategies for sampling the model parameter space could be developed; for example via Markov chain Monte Carlo techniques.

\section{Acknowledgment}

This work was supported by the Engineering and Physical Sciences Research Council and the Research Councils UK Digital Economy Programme, under grant EP/I016058/1. DJH was also supported by a Fellowship from the Leverhulme Trust. 


\section{References}

1. Bullmore, E., Sporns, O.: Complex brain networks: graph theoretical analysis of structural and functional systems. Nature Reviews Neuroscience 10, 186-198 (2009)

2. Easley, D., Kleinberg, J.: Networks, Crowds, and Markets: Reasoning About a Highly Connected World. Cambridge University Press (2010)

3. Estrada, E.: The Structure of Complex Networks. Oxford University Press, Oxford (2011)

4. Granovetter, M.: The Strength of Weak Ties. The American Journal of Sociology 78(6), 13601380 (1973)

5. Grindrod, P., Higham, D.J.: Evolving graphs: Dynamical models, inverse problems and propagation. Proceedings of the Royal Society, Series A 466, 753-770 (2010)

6. Grindrod, P., Higham, D.J.: Bistable evolving networks (To appear in Internet Mathematics) (2012)

7. He, Y., Chen, Z.J., Evans, A.C.: Small-world anatomical networks in the human brain revealed by cortical thickness from MRI. Cerebral Cortex 17(10), 2407-2419 (2007)

8. Hirotugu, A.: A new look at the statistical model identification. IEEE Transactions on Automatic Control 19(6) (1974)

9. Holme, P., Saramäki, J.: Temporal networks. Physics Reports 519, 97-125 (2012)

10. Hu, H., Wang, X.: Evolution of a large online social network. Physics Letters A 373(1213), 1105-1110 (2009)

11. Jackson, M.O.: Networks and economic behavior. The Annual Review of Economics 1, 489513 (2009)

12. Leskovec, J., Backstrom, L., Kumar, R., Tomkins, A.: Microscopic evolution of social networks. In: Y. Li, B. Liu, S. Sarawagi (eds.) KDD, pp. 462-470. ACM (2008)

13. Liu, Y., Liang, M., Zhou, Y., He, Y., Hao, Y., Song, M., Yu, C., Liu, H., Liu, Z., Jiang, T.: Disrupted small-world networks in schizophrenia. Brain 131(4), 945-961 (2008)

14. Mislove, A., Koppula, H.S., Gummadi, K.P., Druschel, P., Bhattacharjee, B.: Growth of the Flickr Social Network. In: Proceedings of the 1st ACM SIGCOMM Workshop on Social Networks (WOSN'08). Seattle, WA (2008)

15. Newman, M.: Networks: An Introduction. Oxford University Press, New York (2010)

16. Rapoport, A.: Spread of information through a population with socio-structural bias. The Bulletin Of Mathematical Biology 15(4), 523-533 (1953)

17. Szell, M., Thurner, S.: Measuring social dynamics in a massive multiplayer online game. Social Networks 39, 313-329 (2010). URL http://arxiv.org/abs/0911.1084

18. Wasserman, L.: All of Statistics: A Concise Course in Statistical Inference. Springer, New York (2004) 